\title{
Gasmischanlage zur automatischen Generierung von VOC-Spurengasen über einen weiten Konzentrationsbereich
}

\author{
Martin Leidinger, Nikolai Helwig, Tilman Sauerwald, Andreas Schütze \\ Lehrstuhl für Messtechnik, Universität des Saarlandes, Saarbrücken \\ Tel.: 0681-302 2235, e-mail: m.leidinger@Imt.uni-saarland.de
}

\section{Kurzfassung}

Es wird eine Laboranlage zur automatischen Generierung von Prüfgasgemischen vorgestellt. In der Anlage werden drei Methoden zur Erzeugung von Spurengasen mit Konzentrationen im ppb- und sub-ppb-Bereich eingesetzt. Die Prüfgase können dabei in einem sehr weiten Konzentrationsbereich zur Verfügung gestellt werden, je nach verwendetem Verfahren. Die Anlage kann darüber hinaus Sauerstoffpartialdruck und relative Gasfeuchte in einem weiten Bereich einstellen.

Die Anlage ist vollständig softwaregesteuert und ermöglicht die einfache Durchführung komplexer Gastests. Die Konzentration an organischen Verunreinigungen wurde mit Hilfe von ex-situ GC/MS Messungen bestimmt und liegt unterhalb von $10 \mu \mathrm{g} / \mathrm{m}^{3}$.

\section{Einleitung}

In der Gasmesstechnik ist eine vielseitige und reproduzierbare Generierung von Prüfgasen unerlässlich. Je nach Anwendungsszenario müssen vielfältige Parameter des zu vermessenden Gases kontrollierbar und variabel sein, dazu gehören Prüfgaskonzentration(en), Gasfeuchte und Flussmenge. Diese Größen müssen zudem kontinuierlich überwacht werden, um die Nachvollziehbarkeit der Gassensorsignale zu gewährleisten. 
Zum Abdecken dieser Anforderungen wurde eine Gasmischanlage (GMA) entwickelt und aufgebaut, die insbesondere auf die Generierung von Prüfgasen aus flüchtigen organischen Verbindungen (Volatile Organic Compounds, VOCs) im ppb- und sub-ppb-Bereich optimiert ist [1].

\section{Anlagenkonzept}

Abb. 1 zeigt den prinzipiellen Aufbau der Anlage.

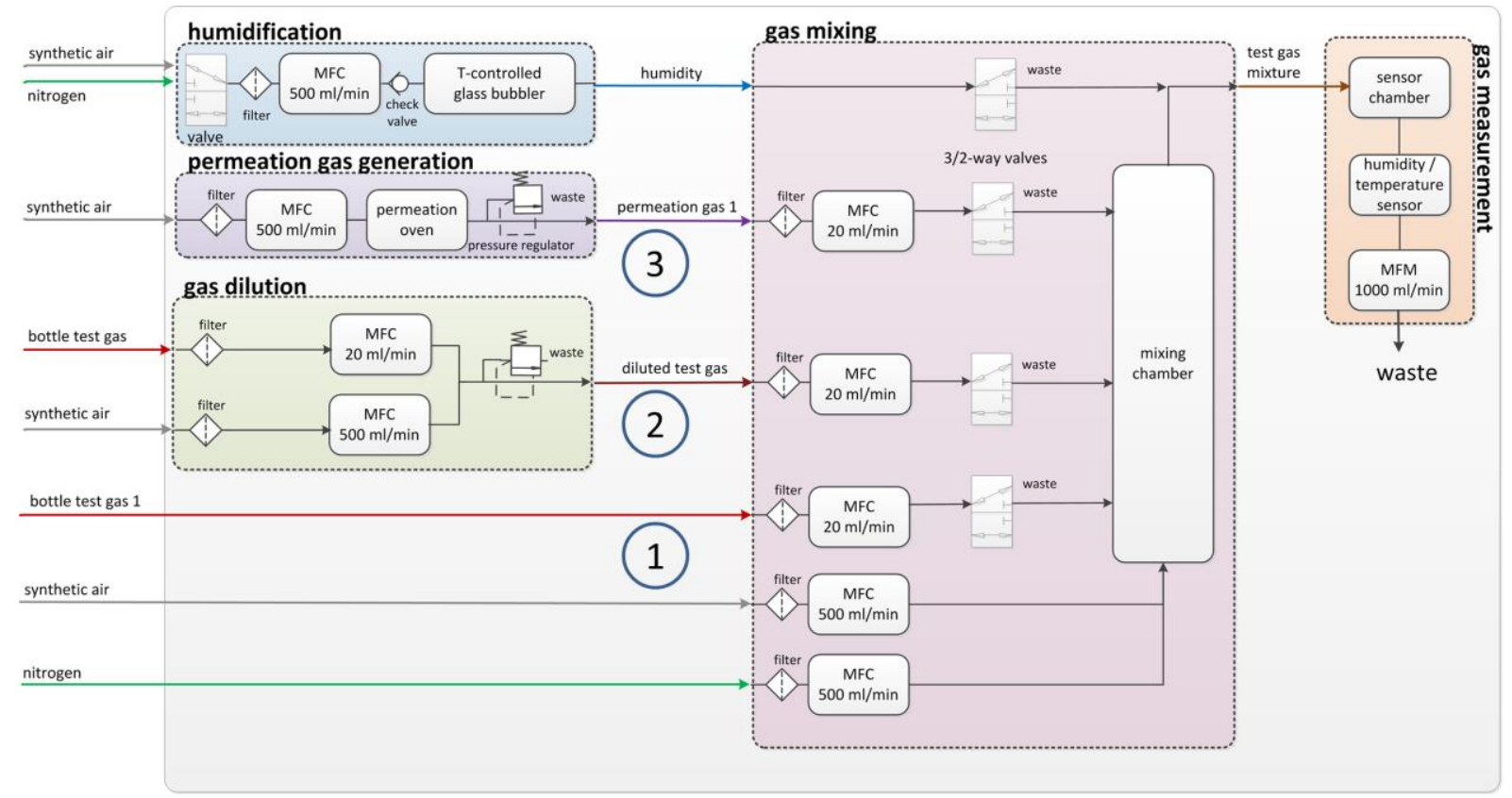

Abb. 1: Schema der Anlage mit den drei verschiedenen Methoden der Prüfgasgenerierung, der Gasmischung und der Gasmessung [1]

In der GMA werden mehrere Prüfgasströme in einen Trägerstrom (typisch 100$500 \mathrm{ml} / \mathrm{min}$ ) aus trockener Luft oder Stickstoff (je in Reinheit 5.0) gemischt. Jeder Prüfgasstrom kann einzeln per 3/2-Wege-Ventil in den Hauptstrom zugeschaltet werden. Nach der Zumischung der Prüfgase (Maximalfluss jeweils $20 \mathrm{ml} / \mathrm{min}$ ) zum trockenen Trägergas wird der feuchte Anteil des Trägergasstroms zugemischt (ebenfalls Luft oder Stickstoff). Das Gas wird dafür durch eine temperierte Waschflasche geleitet und damit feuchtegesättigt. Somit ist eine Feuchte des Gesamtgases von $0 \%$ bis ca. $95 \%$ einstellbar. Über die Nutzung von Stickstoff als Anteil des Trägergases kann der Sauerstoffgehalt des Testgases reduziert werden, um z.B. Verbrennungsabgase zu simulieren. 
Hinter der Gasmischung sind schließlich die Sensorkammern eingerichtet, in denen die zu charakterisierenden Gassensoren in den Testgasstrom eingebracht werden. Weiterhin werden Temperatur und relative Feuchte des Gases gemessen sowie der Gesamtfluss mit einem Massenflussmesser (MFM) überprüft.

\section{Prüfgasgenerierung}

Eine Methode zur rückführbaren Herstellung von Prüfgasgemischen ist die Dosierung von Masseströmen mittels Massenflussreglern (MFC). Dabei wird typischerweise ein Prüfgas einem Trägergasstrom beigemischt, um eine variable Konzentration einzustellen. Nachteilig an dieser Lösung sind der eingeschränkte Dynamikbereich der MFCs sowie die Schwierigkeit, kleinste Konzentrationen bis in den sub-ppb Bereich zuverlässig bereitzustellen.

Massenflussregler arbeiten zuverlässig von $100 \%$ bis ca. $5 \%$ ihres Flussbereichs, sie können somit nur etwa einen Faktor 20 als Dynamikbereich abdecken.

Das Erzeugen sehr kleiner Zielgaskonzentrationen aus typischen Druckgasflaschen ist zudem problematisch, da in diesen Flaschen immer eine Verunreinigung von bis zu 10 ppm enthalten ist. Das Zielgas sollte deswegen in deutlich höherer Konzentration (mind. 50 ppm) in der Flasche vorhanden sein, was die Erzeugung von Spurengaskonzentration mit einfacher MFC-Anordnung beschränkt.

\subsection{Einstufige Mischung von Prüfgasen aus Druckgasflaschen}

Die angesprochene einfache Methode mit einem Massenflussregler wird in der Anlage für Prüfgaskonzentrationen im ppm-Bereich genutzt. Hier wird das Gas direkt aus der Gasflasche über einen $20 \mathrm{ml} / \mathrm{min}$ MFC in den Trägergasstrom dosiert, siehe Abb. 1, Markierung 1. Durch die Software der Anlage wird dabei die Änderung des Gesamtgasstroms durch Reduzierung des trockenen Trägergasstroms automatisch korrigiert, um Temperaturänderungen der Sensoren zu vermeiden, die Gasfeuchte aber konstant zu halten. Bei einem 
Gesamtfluss von $200 \mathrm{ml} / \mathrm{min}$ ist mit dieser Methode ein Verdünnungsbereich von 1:10 bis 1:200 möglich.

\subsection{Zweistufige Mischung von Prüfgasen aus Druckgasflaschen}

Die hier vorgestellte Gasmischanlage unterstuitzt neben der direkten einstufigen Mischung zusätzlich eine zweistufige Mischung, in der eine zusätzliche Verdünnungsstufe einen wesentlich größeren Dynamikbereich und damit eine sehr viel stärkere Verdünnung erlaubt. Mit der hier genutzten MFC-Anordnung (Abb. 1, Markierung 2) wird in der Vorverdünnungsstufe ein Dynamikbereich von 500 erreicht, so dass die Zielgaskonzentration bei gleichbleibendem Trägergasfluss um einen Faktor 10.000 variiert werden kann. Die erreichbare Verdünnung des Zielgases ist abhängig vom Trägergasstrom und beträgt z.B. bei einem Gesamtfluss von $200 \mathrm{ml} / \mathrm{min}$ 1:10 bis 1:100.000. Somit lassen sich aus Flaschengasen mit Konzentrationen von $50 \mathrm{ppm}$ (z.B. $61 \mathrm{mg} / \mathrm{m}^{3}$ bei Formaldehyd) Spurengase bis minimal $0,5 \mathrm{ppb}$ generieren $\left(0,61 \mu \mathrm{g} / \mathrm{m}^{3}\right.$ Formaldehyd).

\subsection{Mittels Permeation erzeugte Prüfgase}

Eine weitere in der Anlage genutzte Variante der Prüfgaserzeugung nutzt Permeationsröhrchen in beheizten Permeationsöfen, in denen jeweils die Zielsubstanz aus einem Teflonröhrchen in den Gasstrom diffundiert. Die Diffusionsrate ist dabei über die Temperatur des Ofens über einen weiten Bereich einstellbar. Die Rückführbarkeit ist bei dieser Methode durch den Massenverlust der Röhrchen über die Zeit gewährleistet. Auch die Testgase aus den Öfen werden beim Zudosieren in den Hauptgasstrom einstufig verdünnt, siehe Abb. 1, Markierung 3. Genutzt wird diese Methode z.B. bei Substanzen, die einen zu geringen Dampfdruck zur sinnvollen Nutzung einer Gasflasche aufweisen. 


\section{Hardware}

Abb. 2 zeigt die Vernetzung aller Hardware-Komponenten der Gasmischanlage.

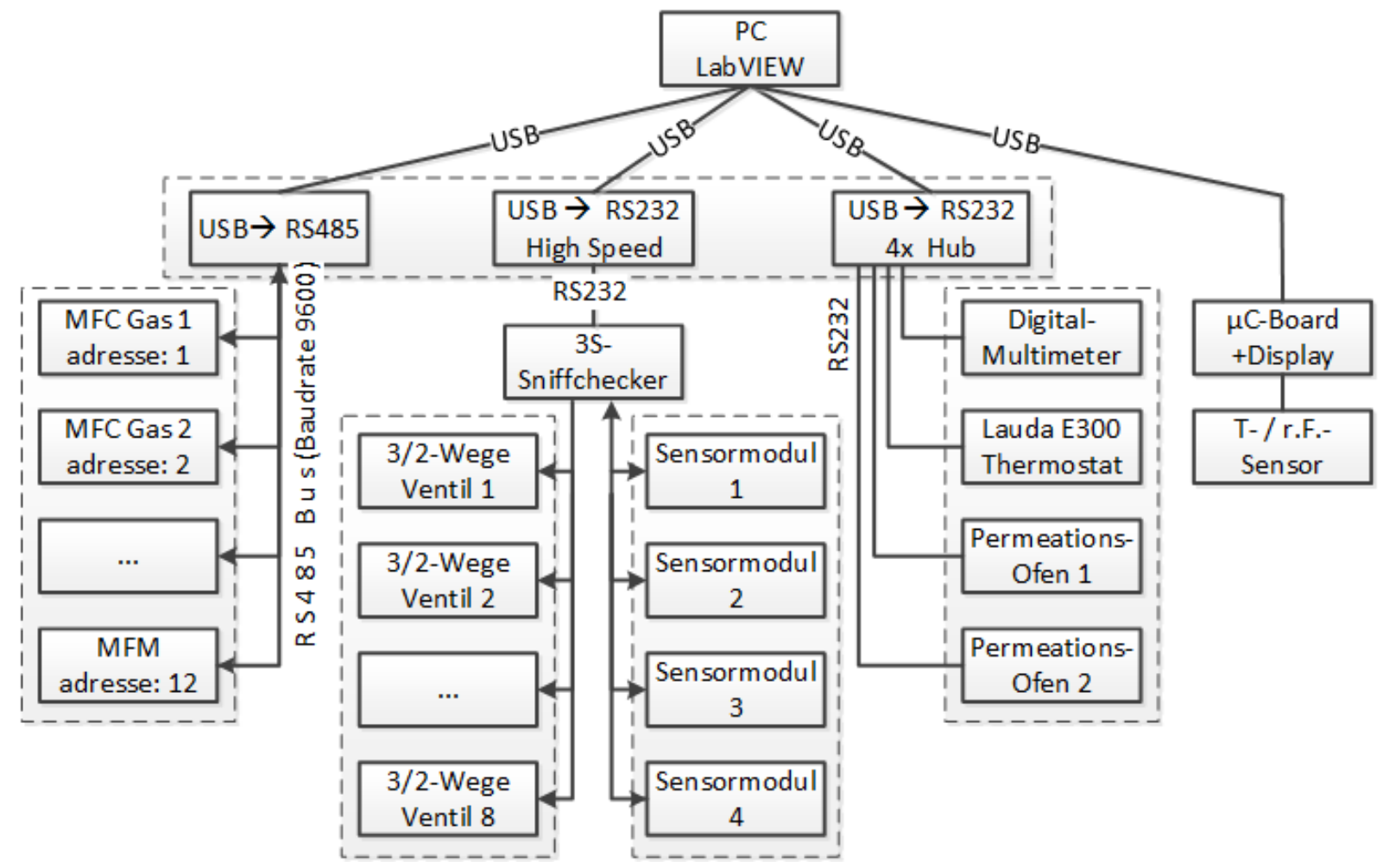

Abb. 2: Hardware-Komponenten der Gasmischanlage

Alle Komponenten des Systems werden von einem zentralen PC aus gesteuert, auf dem gleichzeitig die Datenerfassung erfolgt. Die MFCs sind über einen gemeinsamen RS485-Bus angesteuert. Die Ansteuerung der Ventile und der Gassensoren übernimmt eine „Sniffchecker"-Plattform (3S $\mathrm{GmbH}$, Saarbrücken), die auch für die Synchronisierung in Echtzeit zuständig ist. Mit dieser können bis $\mathrm{zu}$ vier Gassensoren unabhängig voneinander im temperaturzyklischen Betrieb angesteuert und ausgelesen werden [2][3], das Schalten der Ventile zur Beimischung der Prüfgase erfolgt ebenfalls durch diese Elektronik. Weitere Peripherie-Geräte sind ein 10-Kanal-Digitalmultimeter zur Messung analoger Signale (z.B. direkt gemessener Gassensoren), ein Thermostat zur Temperierung des Wasserbads für die Gasbefeuchtung sowie die Permeationsöfen. Alle Geräte sowie die Bussysteme sind direkt oder über entsprechende Konverter per USB an den Rechner angeschlossen. Eine weitere über USB angeschlossene Elektronik liest den Temperatur-/Feuchte-Sensor aus und gibt Informationen zum Betriebszustand der GMA auf einem Display aus. 


\section{Software}

Zur Steuerung der Anlage und zur Konfiguration von Messungen wurde eine Software basierend auf LabVIEW (National Instruments, USA) entwickelt.

Der Benutzer gibt die gewünschten Parameter des Gesamt-Prüfgasgemischs vor (Gaskonzentrationen, Sauerstoffgehalt, relative Feuchte, Gesamtfluss), die Konfiguration der MFCs erfolgt automatisch per Software. Das Erstellen komplexer Gasprofile ist über eine einfache Eingabemaske möglich, in der der Benutzer die gewünschte Gaskonfiguration abschnittsweise festlegt. Pro Fenster werden die Dauer des Abschnitts, die Gaskonzentrationen und die Ventilstellungen eingegeben. Über Schleifen lassen sich schnell komplexe Abläufe festlegen, so kann z.B. ein eingegebenes Gasprofil rasch mit mehreren Gasfeuchten wiederholt werden.

Abb. 3 zeigt ein Beispiel-Testgasprofil, das mit der Anlage erstellt wurde. Hier wurden drei Spurengase (Formaldehyd, Benzol, Naphthalin) in je fünf Konzentrationen bei zwei Luftfeuchten generiert.

\section{Konzentrationsablauf (in \% von Maximalkonzentration)}

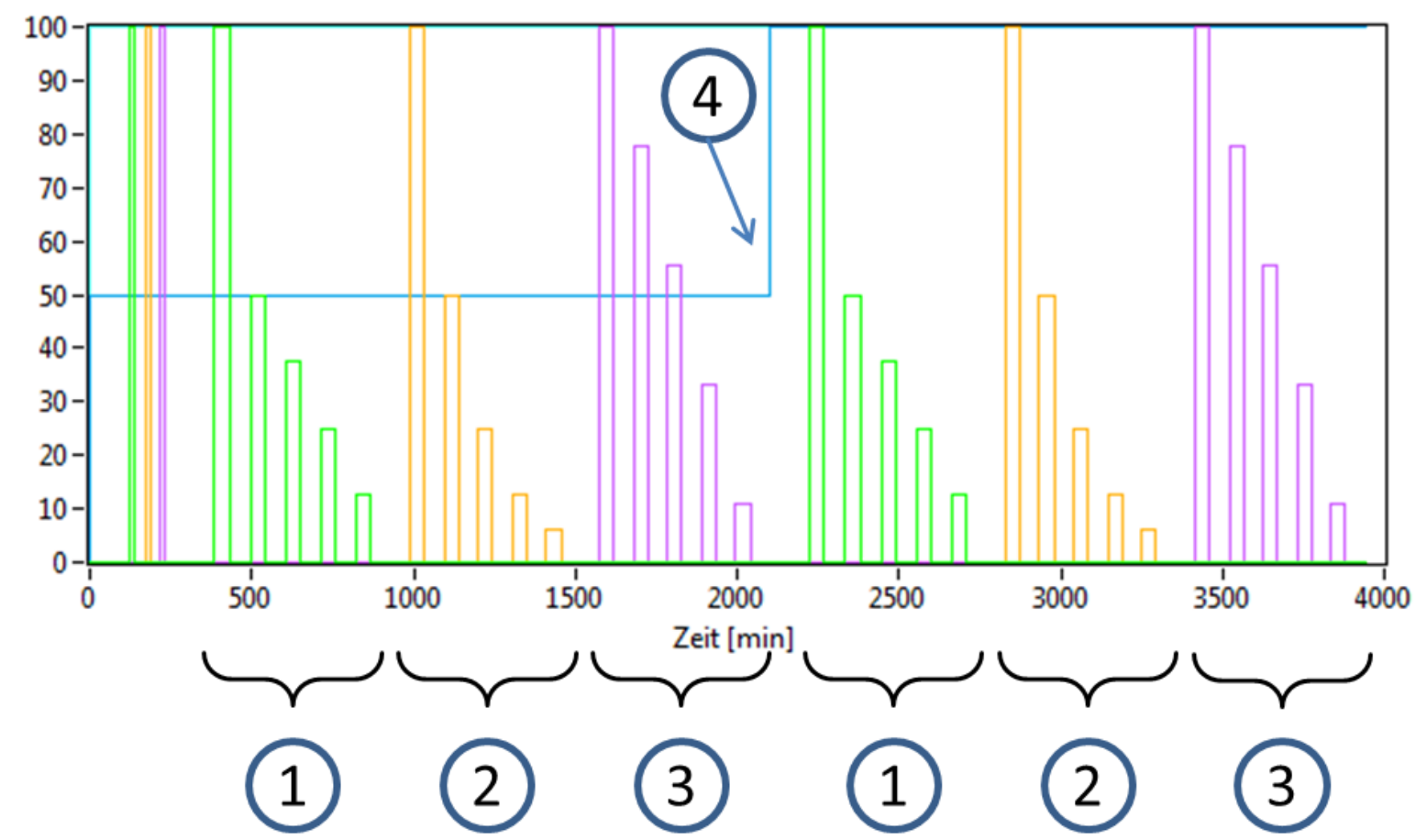

Abb. 3: Beispiel-Gaskonzentrationsprofil. Die Werte sind auf die maximale Konzentration des jeweiligen Gases skaliert. (1): Formaldehyd, $200 \mathrm{ppb}\left(123 \mu \mathrm{g} / \mathrm{m}^{3}\right)$; (2): Benzol, 4,5 ppb $\left(14 \mu \mathrm{g} / \mathrm{m}^{3}\right)$; (3): Naphthalin, $40 \mathrm{ppb}\left(210 \mu \mathrm{g} / \mathrm{m}^{3}\right) ;(4)$ : rel. Luftfeuchte, $40 \%$ 
Alle Benutzereingaben werden direkt auf Plausibilität geprüft; bei nicht durchführbaren Eingaben wird eine Fehlermeldung ausgegeben und der Start der Messung verhindert. Alle relevanten Daten wie Sensorsignale, MFCFlusswerte und Ventilstellungen werden von der Software in einer GesamtMessdatei gespeichert.

\section{Validierung für kleine VOC-Konzentrationen}

Zunächst wurde mit analytischen Messungen (GC/MS) die Reinheit der Anlage untersucht. Es wurden Gasproben an verschiedenen Stellen der Anlage entnommen. Dabei wurden Verunreinigungen von einigen $\mu \mathrm{g} / \mathrm{m}^{3}$ (entspricht wenigen ppb) festgestellt, hauptsächlich durch eine Substanz (siehe Tabelle 1); Ursprung der Verunreinigung war einer der Massenflussregler. Nach einem halben Jahr Betrieb der Anlage wurden die Messungen wiederholt, wobei eine TVOC-Verunreinigung unter $10 \mu \mathrm{g} / \mathrm{m}^{3}$ festgestellt wurde (Tabelle 1, letzte Spalte); die Verunreinigung wurde also zwischenzeitlich ausgespült.

Tabelle 1: Auszug aus der GC/MS-Analyse der aus der GMA strömenden Luft nach Aufbau der Anlage $\left(c_{1}\right)$ [4] und nach einem halben Jahr Betrieb $\left(c_{2}\right)$; (n.d.: nicht detektierbar)

\begin{tabular}{|l|l|l|l|l|}
\hline Compound & CAS number & $\mathbf{c}_{1}\left(\boldsymbol{\mu g} / \mathbf{m}^{\mathbf{3}}\right)$ & $\mathbf{c}_{1}(\mathbf{p p b})$ & $\mathbf{c}_{2}\left(\boldsymbol{\mu g} / \mathbf{m}^{\mathbf{3}}\right)$ \\
\hline Benzol & $71-43-2$ & 0.17 & 0.053 & n.d. \\
\hline Toluol & $108-88-3$ & 0.06 & 0.016 & n.d. \\
\hline Naphthalin & $91-20-3$ & 0.24 & 0.046 & n.d. \\
\hline $\begin{array}{l}\text { Bicyclo[2.2.1]heptan, } \\
\text { 2-chloro-2,3,3-trimethyl- }\end{array}$ & $465-30-5$ & 16.2 & 2.6 & n.d. \\
\hline TVOC & & $\mathbf{2 4 . 2}$ & & $\mathbf{8 , 3 6}$ \\
\hline
\end{tabular}

Ein weiteres Problem sind Verschleppungseffekte, die insbesondere bei weniger flüchtigen Gasen, die mittels Permeation erzeugt werden wie z.B. Naphthalin, auftreten können. Hierbei bleibt das Gas auch nach Entfernen des Permeationsröhrchens an den Oberflächen der Anlage haften (MFCs, Verrohrung) und wird erst nach einiger Zeit komplett ausgespült.

Die Funktionalität der Anlage wurde durch die Generierung von Testgasen mit verschiedenen VOCs mit Konzentrationen im ppb und sub-Bereich verifiziert [4]. Auch die weiteren einstellbaren Größen (Gesamtfluss, 
Sauerstoffgehalt, Feuchte) wurden in verschiedenen Testmessungen variiert und die korrekte Funktion der Anlage überprüft.

\section{Fazit}

Die entwickelte Gasmischanlage stellt ein vielseitiges Werkzeug zur Gassensorcharakterisierung dar. Mit der Methode der Gasvorverdünnung kann ein sehr hoher Dynamikbereich der Gaskonzentrationen erzielt werden. Durch die variable Feuchte und den absenkbaren Sauerstoffgehalt lässt sich eine Vielzahl von möglichen Testszenarien abdecken [4][5][6]. Die Software erlaubt einfaches Erstellen komplexer Gasprofile und integrierte Speicherung aller relevanten Daten.

Die intrinsische Verunreinigung der Anlage konnte auf einen konstanten Wert unter $10 \mu \mathrm{g} / \mathrm{m}^{3}$ (ca. 2-3 ppb) gesenkt werden, wodurch Messungen mit Gaskonzentrationen im ppb-Bereich durchführbar sind. Es können jedoch Verschleppungseffekte auftreten, was bei der Erstellung der Testgasprofile vom Benutzer $\mathrm{zu}$ beachten ist. Mögliche Methoden, um diesem Nachteil entgegenzuwirken, sind Ausheizen der gasberührenden Komponenten oder Beschichten der entsprechenden Oberflächen.

\section{Danksagung}

Diese Arbeiten wurden durchgeführt im Rahmen des MNT-ERA.net-Projekts VOC-IDS, gefördert vom Bundesministerium für Bildung und Forschung (Förderkennzeichen 16SV5480K). Die GC/MS-Analysen wurden durchgeführt von Dr. Gabriela Ventura am IDMEC-FEUP, Porto, Portugal.

\section{Literatur}

[1] N. Helwig, M. Schüler, C. Bur, A. Schütze and T. Sauerwald, "Gas mixing apparatus for automated gas sensor characterization", Meas. Sci. Technol. 25 (2014) 055903 (9pp) 
[2] A.P. Lee, B.J. Reedy, „Temperature modulation in semiconductor gas sensing", Sensors and Actuators B 60 (1999), pp. 35-42

[3] A. Gramm, A. Schütze, „High performance solvent vapor identification with a two sensor array using temperature cycling and pattern classification", Sensors \& Actuators B 95 (2003) pp. 58-65

[4] M. Schüler, N. Helwig, G. Ventura, A. Schütze and T. Sauerwald, "Detecting Trace-Level Concentrations of Volatile Organic Compounds with Metal Oxide Gas Sensors", IEEE Sensors Conference 2013; Baltimore, MD, USA, Nov. 03 - 06, 2013

[5] C. Bur, M. Andersson, A. Lloyd Spetz and A. Schütze, "Detecting Volatile Organic Compounds in the ppb Range with Gas Sensitive Platinum gate SiC-Field Effect Transistors", IEEE Sensors Journal, online prepublication May 23, 2014, doi: 10.1109/JSEN.2014.2326693

[6] M. Leidinger, T. Sauerwald und A Schütze: „Optimierter dynamischer Betrieb und multivariate Signalauswertung zum selektiven Nachweis von VOC im ppb-Bereich“, 17. ITG / GMA Fachtagung Sensoren und Messsysteme 2014, Nürnberg, 03./04. Juni 2014, ITG Fachbericht 250, VDE Verlag, 2014, ISBN 978-0-8007-3622-5 